\title{
Multiscale Twin Hierarchy in NiMnGa Shape Memory Alloys with Fe and Cu
}

\author{
Rozaliya I. Barabash ${ }^{1 *}$, Oleg M. Barabash ${ }^{2}$, Dmitry Popov $^{3 * *}$, Guoyin Shen ${ }^{3}$, Changyong Park, ${ }^{3}$ \\ Wenge Yang ${ }^{4}$ \\ ${ }^{1}$ Oak Ridge National Laboratory, Oak Ridge, TN \\ ${ }^{2}$ The University of Tennessee \\ ${ }^{3}$ HPCAT, Geophysical Laboratory, Carnegie Institution of Washington, Argonne, IL 60439 \\ ${ }^{4}$ Center for High Pressure Science and Technology Advanced Research (HPSTAR), Shanghai \\ 201203, P. R. China
}

\begin{abstract}
X-ray microdiffraction and scanning electron microscopy studies reveal 10M martensitic structure with a highly correlated multiscale twin hierarchy organization in $\mathrm{NiMnGaFeCu}$ shape memory alloys. High compatibility is found at the twin interfaces resulting in a highly correlated twinned lattice orientation across several laminate levels. The lattice unit cell is described as monoclinic I-centered with $a=4.28 \AA, b=4.27 \AA, c=5.40 \AA, \gamma=78.5^{\circ}$. The modulation is found parallel to the $b$ axis. Thin tapered needle-like lamellae and branching are observed near the twin boundaries.
\end{abstract}

\section{Keywords: Shape memory alloys; X-ray microdiffraction; Twin boundaries; Scanning electron microscopy; Multiscale structure.}

Corresponding authors e-mail: *<rbarabas@utk.edu $>$; **dpopov@ carnegiescience.edu 


\section{Introduction}

Ferromagnetic off-stoichiometric Heusler-type NiMnGa-based (NMG) alloys recently attracted a lot of attention due to the unique combination of different properties, such as shape memory and magnetic memory, magnetocaloric effect, and large magnetoresistence [1-6]. These alloys have high potential for practical applications in actuators, sensors, and energy harvesters [5, 7]. With temperature decrease the NMG alloys undergo the $1^{\text {st }}$ order displacive (martensitic) transformation from L2 $2_{1}$ structure to a tetragonal, orthorhombic or monoclinic martensitic phase [8-10]. In the first approximation, it was considered that martensite in NMGs can be described in tetragonal approximation. However, soon it became clear that the martensite in NMGs requires a description beyond the tetragonal approximation $[11,12]$. Later several distinct martensite structures were observed in NMGs: seven (14M) modulated martensite [3, 13], five (10M) modulated martensite [14-16], nonmodulated martensite [17]. It was suggested that the shape memory effect is largely determined by the arrangement, type and movement of the twin boundaries (TBs) in NMG alloys [18-22]. The minimal stress needed to move a TB determines the critical twinning stress. Two types of the TBs were reported in 10M martensite: type I and type II TBs $[15,23]$. Type I TBs have a twinning stress $\sim 1 \mathrm{MPa}$ at room temperatures, while type II TBs usually have a much smaller twinning stress $\sim 0.2 \mathrm{MPa}$. The twinning stress for the type I TBs is strongly temperature dependent, while for the type II TBs it is very weakly temperature dependent $[23,24]$. It was found that at least two levels of "lamination" are possible in the NMG crystals. There is still an open question "how such microstructures can be connected compatibly over a single interface?" [23]. 
Phase transition temperature in ferromagnetic NiMnGa-based alloys can be modified by the magnetic field, external strain field and alloy composition. It was noticed that off-stoichiometric alloys often demonstrate higher shape memory effect than stoichiometric $\mathrm{Ni}_{2} \mathrm{MnGa}$ alloys [2530].

Commensurate as well as incommensurate types of modulated martensite structures were derived from experimental data [31-33]. It was noted, that there is a controversy between the conclusions of [31] and the results of [33]. Specifically [31] emphasized that "The change of the composition from stoichiometry $\left(\mathrm{Ni}_{2} \mathrm{MnGa}\right)$ could imply a different behaviour of the modulation amplitudes of atomic sites differently occupied by chemical species." These authors argue that: "The historical classification of martensitic modulated structures based on the number of observed satellites must be reconsidered". For many applications the shape memory materials should be able to operate at elevated temperatures above $130^{\circ} \mathrm{C}$ [34]. The operating temperatures of shape memory alloys are determined by their Curie and structural phase transition temperatures. In the same time for different magnetocaloric applications the operating temperatures should be close to ambient. It was noticed that additional elements may essentially modify the transition temperature and enhance both shape memory and caloric effects. In particular, additions of Fe [34-37] and $\mathrm{Cu}[38,39]$ appeared very effective to shift the transition temperature and modify shape memory and magnetocaloric effects [40]. While in stoichiometric and off- stoichiometric ternary NMGs the martensite structures were studied relatively well, the structure of NMGs additionally doped with $\mathrm{Fe}$ and $\mathrm{Cu}$ is still poorly understood. Therefore several alloys with different additions of $\mathrm{Fe}$ and $\mathrm{Cu}$ were prepared and compared to ternary NiMnGa alloys with different Ni:Mn:Ga ratio [40]. The alloy with the highest martensitic transition temperature was chosen for this study. 
In this letter we report the multiscale TB hierarchy in the martensite structure of the NMG alloy with additions of $\mathrm{Fe}$ and $\mathrm{Cu}$ with microdiffraction and scanning electron microscopy to explore the micromechanism of twinning effects.

\section{Materials and Experimental procedures}

The polycrystalline $\mathrm{Ni}-\mathrm{Mn}-\mathrm{Ga}-\mathrm{Fe}-\mathrm{Cu}$ alloy composition corresponds to the following stoichiometry: $2.3 / 0.7 / 0.96 / 0.03 / 0.03$. The martensite transition temperature of the alloy is 619 K. The ingot rod with $12.7 \mathrm{~mm}$ diameter and $19.0 \mathrm{~mm}$ length was prepared by arc melting in argon atmosphere. It was re-melted four times, annealed at $1100 \mathrm{~K}$ in evacuated quartz ampoules in high purity helium environment for $72 \mathrm{~h}$. The sample is similar to the "sample \#3" described by [41].

The martensite structure was studied by X-ray microdiffraction and Electron Back Scattering Diffraction (EBSD, actually SEM can give this kind of high resolution backscattering image). EBSD measurements were performed using a Philips XL30 field-emission scanning electron microscope equipped with a DigiView camera. For EBSD measurements the ingot was cut perpendicular to the growth direction of the rod. Sample surface was initially grinded with 2400 grit $\mathrm{SiC}$ paper, and then vibrationally polished with a slurry of $0.3 \mu \mathrm{m} \mathrm{Al}_{2} \mathrm{O}_{3}$ and with a colloidal suspension of $\sim 20 \mathrm{~nm} \mathrm{SiO}_{2}$ in sequence. To obtain a sample for X-ray microdiffraction study, first a disk with a thickness of $0.5 \mathrm{~mm}$ was cut from the rod, after that a thin needle was cut by spark erosion.

The needle-shaped sample with around $0.5 \mathrm{~mm}$ thickness was studied by X-ray diffraction technique using monochromatic X-ray beam at 16BMD, and with white beam at 16BMB 
beamlines of Advanced Photon Source (APS). X-ray monochromatic beam of $0.4133 \AA$ wavelength was focused down to $15 \mu \mathrm{m}$ vertically and $5 \mu \mathrm{m}$ horizontally using KB-mirrors. The sample rotated in step of $1^{\circ}$ collecting X-ray diffraction pattern with MAR345 IP area detector during each step of rotation. 80 frames have been collected in total. The diffraction images have multiple spots from multiple crystalline grains present in the beam simultaneously; however a single grain which has dominant contribution to the diffraction patterns could be identified. Reflections from this single grain (about 100 distinguishable diffraction spots) have been selected using a specifically developed software and the final refinement of its unit cell parameters was done with the XDS package [42].

$\mathrm{X}$-ray Laue micro diffraction was performed in transmission geometry in order to distinguish crystalline twin domains with a better angular resolution. White beam within the 5$70 \mathrm{keV}$ energy range was focused down to $\sim 10 \times 10 \mu \mathrm{m}^{2}$ using KB-mirrors. Diffraction patterns were recorded using MARCCD area detector with 16bit readout and $80 \times 80 \mu \mathrm{m}^{2}$ pixel size positioned at around $170 \mathrm{~mm}$ from the sample. Sample positioning was done with two horizontal and one vertical translational stages moving in directions perpendicular to each other. The horizontal stages were mounted on top of a rotational stage which made possible measurements at different angular positions. Precision of the sample positioning and eccentricity of the rotation stage were about $1 \mu \mathrm{m}$. An in-line Si 111 channel cut monochromator was used to provide monochromatic beam of $0.3065 \AA$ wavelength, which is readily exchangeable with the white beam setup. The monochromatic beam was exploited for calibration of sample to detector distance and detector tilt with $\mathrm{CeO}_{2}$ powder standard using Fit2d software [43]. Indices of Laue reflections were found from orientation matrix previously determined with this monochromatic beam. Finally orientation matrices of studied crystals have been found more precisely based on 
known indices and positions of white beam reflections using specifically developed software routines. Fit2d peak search feature was used to find centers of diffraction spots. Based on positions and indices of two strong reflections from each individual crystal its orientation matrix have been calculated $[44,45]$, therefore coordinates of other reflections from the same grain were predicted in order to compare them with the experimentally determined ones which in turn allowed to recognize twin domains.

\section{Results and Discussion}

Hierarchical twin microstructure in the Ni-Mn-Ga-Fe-Cu alloy is clearly demonstrated by SEM (Figs. 1 and 2). At the mesoscale, the twin structure consists of alternating, so-called, "compound" twins with an average thickness of $\sim 10 \mu \mathrm{m}$ (Fig.1a). Higher magnification reveals the next structural level of twins within these compound twins - so called "laminate" (Fig. 1b). At the grain boundaries between two different laminates a lot of branching is observed with the formation of thin tapering needles (Figs.1 c and d). These structural peculiarities confirm the microstructural model for macro-twin interfaces that was recently suggested by [23]. Within the framework of their model, the "minor variant in each laminate forms thin, tapering needles ending at the interface", which exactly corresponds to the features observed in Figs.1 c, d. The thin tapering needles are formed at the TB due to their higher energy for nucleation at the front of the moving TB, than at the side of the TB. According to [46] this "is leading to the long and narrow shape of lamellae. At the atomistic scale, the needle shape is formed by steps on the twin wall". Figs. 2 a, b show a compatible micro-twin boundary with continuity at the twin interface. Further increase of magnification reveals the next finer level of the twin colonies with the size of the twin lamellae below $100 \mathrm{~nm}$ (Fig. 2 c, d). While [11, 23] observed two levels of twin laminate at the scale higher than $10 \mu \mathrm{m}$, the next 4 higher levels of laminate reaching from 10 
$\mu \mathrm{m}$ to $100 \mathrm{~nm}$ are found. This agrees with the comment by [23] that "the presence of fine laminates (possibly higher orders) close to the interface does not contradict their concept". The characteristic length scale of the laminate, starting from $10 \mu \mathrm{m}$ and reaching up to $100 \mathrm{~nm}$ confirms the suggestion of the [46] that there may be some internal length scale of the energy landscape in the shape memory material which influences the motion of the TB.

Unit cell parameters were determined by X-ray diffraction. Using the monochromatic beam diffraction, about 100 reflections collected from a single grain yielded orthorhombic unit cell with the following parameters: $a=4.276(5) \AA, b=20.93(2) \AA, c=5.397(7) \AA$. As the first approximation, the $b$-axis dimension indicates the formation of the 5 modulated martensite (M10) in the Ni-Mn-Ga-Fe-Cu alloy. Examination of the same grain of the sample with the white beam provided much better angular resolution of inter crystalline orientation and indicated that the beam was probing a twinned laminate consisting of colonies with two twin unit cell orientations and related to each other by mirror plane (Fig. 3 left). The lattice is modulated along the $b$ axis of the above orthorhombic unit cell (Fig. 3 right). If the crystal lattice of one colony is reflected by this plane, then the resulting lattice will deviate from the second colony lattice only by $\sim 0.3^{\circ}$. (calculated from the orientation matrices of the colonies). Angular step used for the diffraction measurements with the monochromatic beam was much larger than this deviation overlap of the reciprocal lattices, therefore the twinned colonies were always observed within mutual pseudo orthorhombic reciprocal space lattice. Unit cell parameters of the twinned lamellae have been calculated based on the pseudo orthorhombic cell. They turned out to be Icentered monoclinic lattice: $a=4.28 \AA, b=4.27 \AA, c=5.40 \AA, \gamma=78.5^{\circ}$.

In Fig. 4, two indexed Laue diffraction patterns are shown. Average deviation between the predicted and observed positions of reflections are within 3 pixels limit. Only a few 
reflections exhibit such deviation up to 7 pixels which can be explained by the following reasons. First of all, unit cell of each separate colony in the pseudo orthorhombic setting may not have precise orthorhombic matrix as it was assumed for the calculations of the reflections positions. Another possible reason is that the diffraction spots exhibit some broadening and diffuse tails which introduced some uncertainty to the determination of their positions. Some reflections were oversaturated, so their precise positions were not available. On the other hand, the predicted positions of reflections from the opposite twin orientation reflected in the plane of twinning exhibit much higher deviation from the observed positions: 7-9 pixels in average and up to 15 pixels. Four such predicted positions are exemplary shown on Fig. 4 in comparison with the predicted positions of the domains themselves. Reflections with $\mathrm{h}=5$ exhibit splitting because each of the twin orientations has such a reflection, which clearly indicates that all these reflections cannot be indexed assuming only one lattice orientation.

The observations from Laue microdiffraction are supported by the above SEM results, which show that the martensite structure consists of multiscale hierarchy of twin colonies (laminate). With the beam size of $\sim 10$ micrometers for Laue microdiffraction, being much higher than the thickness of the individual lamellae within the twinned lattices, the beam is always probing the multiscale twinned laminate within one grain (Figs $1 \mathrm{~b}$ and $2 \mathrm{a}, \mathrm{b}$ ). Therefore the reflections from both twinned lattice orientations are always simultaneously present at the Laue pattern. In Laue diffraction, the position of the Laue spot at the pattern is determined by the lattice plane orientation. The fact that the whole colony of lamellae diffract almost in the same direction of reciprocal space demonstrates highly coherent orientation between lamellae within the twinned colonies laminate (as seen at the Fig. 2b). The results support the model of 
hierarchically twinned martensite, described by Mullner and collaborators [19-21] at the microscopic and mesoscopic scales.

\section{Conclusions}

X-ray microdiffraction and SEM study support the model of several levels of twin laminate within individual grain of the polycrystalline sample - "twins within twins". Combination of X-ray microdiffraction and SEM shows that the nanolaminate structure is extended to much smaller length scales, than was previously assumed. The Ref. [24] suggested that laminate structure had a length scale up to $25 \mu \mathrm{m}$, and noted that a smaller probe size is needed to identify the features below this length scale. Our observations identify at least 4 levels of twinned laminates reaching from 10 micrometers to $100 \mathrm{~nm}$. High compatibility is found at the twin interfaces resulting in a highly correlated twinned lattices orientation across several laminate levels, which addresses the problem raised by [23] on "how such microstructures can be connected compatibly over a single interface”. X-ray micro diffraction study confirmed the twin laminate structure: two twin orientations have been recognized with an I-centered monoclinic

lattice: $a=4.28 \AA, b=4.27 \AA, c=5.40 \AA, \gamma=78.5^{\circ}$ corresponding to the $10 \mathrm{M}$ martensite with modulation direction along the $b$ direction of the unit cell.

\section{Acknowledgement}

Research sponsored by the Laboratory Directed Research and Development Program of Oak Ridge National Laboratory, managed by UT-Battelle, LLC, for the U. S. Department of Energy. 
Research conducted as part of a user project supported by ORNL's Center for Nanophase Materials Sciences, which is sponsored by the Scientific User Facilities Division, Office of Basic Energy Sciences, U.S. Department of Energy. HPCAT operations are supported by DOE-NNSA under Award No. DE-NA0001974 and DOE-BES under Award No. DE-FG02-99ER45775, with partial instrumentation funding by NSF. The Advanced Photon Source is a U.S. Department of Energy (DOE) Office of Science User Facility operated for the DOE Office of Science by Argonne National Laboratory under Contract No. DE-AC02-06CH11357. 


\section{References}

1. Heczko, O., Magnetoelastic Coupling in Ni-Mn-Ga Magnetic Shape Memory Alloy. Materials Science Forum, 2009. 635: p. 125-130.

2. Ma, J. and I. Karaman, Materials science. Expanding the repertoire of shape memory alloys. Science, 2010. 327(5972): p. 1468-9.

3. Sozinov, A., et al., Giant magnetic-field-induced strain in NiMnGa seven-layered martensitic phase. Applied Physics Letters, 2002. 80(10): p. 1746.

4. Barabash, R.I., et al., In-situ observation of stress-induced stochastic twin boundary motion in off stoichiometric NiMnGa single crystals. Applied Physics Letters, 2013. 103(2): p. 021909.

5. Sharpee, T., M. Dykman, and P. Platzman, Tunneling decay in a magnetic field. Physical Review A, 2002. 65(3).

6. Barabash-Sharpee, T., Dykman, M.I., Platzman, P.M. , Tunneling Transverse to a Magnetic Field and Its Occurrence in Correlated 2D Electron Systems. PRL, 2000. 84(10): p. 2227-2230.

7. Wilson, S.A., et al., New materials for micro-scale sensors and actuators. Materials Science and Engineering: R: Reports, 2007. 56(1-6): p. 1-129.

8. Wang, D.-H., et al., Martensitic transformation and related magnetic effects in $\mathrm{Ni}-\mathrm{Mn}$ based ferromagnetic shape memory alloys. Chinese Physics B, 2013. 22(7): p. 077506.

9. Shapiro, S., et al., Neutron-scattering and electron-microscopy studies of the premartensitic phenomena in NixAl100-x alloys. Physical Review B, 1991. 44(17): p. 9301-9313.

10. Kushida, H., et al., Crystal structure of martensite and intermediate phases in Ni2MnGa studied by neutron diffraction. The European Physical Journal Special Topics, 2008. 158(1): p. 87-92.

11. Straka, L., et al., Highly mobile twinned interface in 10M modulated $\mathrm{Ni}-\mathrm{Mn}-\mathrm{Ga}$ martensite: Analysis beyond the tetragonal approximation of lattice. Acta Materialia, 2011. 59(20): p. 7450-7463.

12. Singh, S., et al., $(3+1) D$ superspace description of the incommensurate modulation in the premartensite phase of Ni2MnGa: a high resolution synchrotron $x$-ray powder diffraction study. J Phys Condens Matter, 2013. 25(21): p. 212203.

13. Sozinov, A., et al., Stress- and magnetic-field-induced variant rearrangement in Ni-MnGa single crystals with seven-layered martensitic structure. Materials Science and Engineering: A, 2004. 378(1-2): p. 399-402.

14. Sozinov, A., et al., Highly mobile type II twin boundary in Ni-Mn-Ga five-layered martensite. Applied Physics Letters, 2011. 99(12): p. 124103.

15. Chulist, R., et al., Characterization of mobile type I and type II twin boundaries in 10M modulated $\mathrm{Ni}-\mathrm{Mn}-\mathrm{Ga}$ martensite by electron backscatter diffraction. Acta Materialia, 2013. 61(6): p. 1913-1920.

16. Chulist, R., et al., Twin boundaries in trained 10M Ni-Mn-Ga single crystals. Scripta Materialia, 2012. 67(4): p. 364-367.

17. J. Pons, V.A.C., R. Santamarta and E. Cesari, Crystal structure of martensitic phases in Ni-Mn-Ga shape memory alloys. Acta Materialia, 2000. 48: p. 3027-3038.

18. Sozinov, L., Lanska, Sodeberg, Ullako and Lindroos. Effect of crystal structure on magnetic-field-induced straine in Ni-Mn-Ga. in SPIE. 2003. 
19. Müllner, P., King, A. H., Deformation of hierarchically twinned martensite. Acta Materialia, 2010. 58(16): p. 5242-5261.

20. Mullner, P., Between microscopic and mesoscopic description of twin-twin interaction. Zeitschrift für Metallkunde, 2006. 97(3): p. 205-216.

21. Mullner, P., Romanov, A. E., Internal Twinning in Deformation Twinning. Acta Mater., 2000: p. 2323-2337.

22. Rolfs, K., Chmielus, M., Wimpory, R. C., Mecklenburg, A., Müllner, P., Schneider, R., Double twinning in Ni-Mn-Ga-Co. Acta Materialia, 2010. 58(7): p. 2646-2651.

23. Seiner, H., L. Straka, and O. Heczko, A microstructural model of motion of macro-twin interfaces in Ni-Mn-Ga 10M martensite. Journal of the Mechanics and Physics of Solids, 2014. 64: p. 198-211.

24. Straka, L., et al., Ni-Mn-Ga single crystals with very low twinning stress. Journal of Physics: Conference Series, 2011. 303: p. 012079.

25. Richard, M.L., et al., Chemical order in off-stoichiometric Ni-Mn-Ga ferromagnetic shape-memory alloys studied with neutron diffraction. Philosophical Magazine, 2007. 87(23): p. 3437-3447.

26. Enkovaara, J., et al., Magnetically driven shape memory alloys. Materials Science and Engineering: A, 2004. 378(1-2): p. 52-60.

27. Entel, P., et al., Shape Memory Alloys: A Summary of Recent Achievements. Materials Science Forum, 2008. 583: p. 21-41.

28. Lázpita, P., et al., Magnetic moment and chemical order in off-stoichiometric Ni-Mn-Ga ferromagnetic shape memory alloys. New Journal of Physics, 2011. 13(3): p. 033039.

29. Chernenko, V.A., et al., Giant two-way shape memory effect in high-temperature Ni-MnGa single crystal. Physics Procedia, 2010. 10: p. 94-98.

30. Barabash, R.I., et al., Structural and dynamical fluctuations in off-stoichiometric NiMnGa shape-memory alloys. Applied Physics Letters, 2014. 104(24): p. 241905.

31. Righi, L., et al., Commensurate and incommensurate "5M" modulated crystal structures in Ni-Mn-Ga martensitic phases. Acta Materialia, 2007. 55(15): p. 5237-5245.

32. Righi, L., et al., Incommensurate modulated structure of the ferromagnetic shapememory Ni2MnGa martensite. Journal of Solid State Chemistry, 2006. 179(11): p. 35253533.

33. Brown, C., Kanomata, Matsumoto, Neumann, Ouladdiaf and Ziebeck, The crystal structure and phase transitions of the magnetic shape memory compound Ni2MnGa. J. Phys.:Condens. Matter, 2002. 14: p. 10159-10171.

34. Guldbakke, J.M., et al., Magnetic, mechanical and fatigue properties of a Ni45.4Mn29.1Ga21.6Fe3.9 single crystal. Scripta Materialia, 2010. 62(11): p. 875-878.

35. Liu, Z.H., et al., Magnetic properties and martensitic transformation in quaternary Heusler alloy of NiMnFeGa. Journal of Applied Physics, 2002. 92(9): p. 5006.

36. Yu, S.Y., et al., Magnetic field-induced martensite-austenite transformation in $\mathrm{Fe}$ substituted NiMnGa ribbons. Scripta Materialia, 2011. 65(1): p. 9-12.

37. Huang, C.H., et al., Fe substitution induced intermartensitic transition and its internal stress dependent transforming behavior in Ni-Mn-Ga based alloy. Journal of Alloys and Compounds, 2013. 581: p. 812-815.

38. Li, P.-P., J.-M. Wang, and C.-B. Jiang, Martensitic transformation in Cu-doped NiMnGa magnetic shape memory alloys. Chinese Physics B, 2011. 20(2): p. 028104. 
39. Roy, S., et al., Delocalization and hybridization enhance the magnetocaloric effect in $\mathrm{Cu}$ doped Ni2MnGa. Physical Review B, 2009. 79(23).

40. Nicholson, D.M., Odbadrakh, Kh, Rusanu, A., Eisenbach, M., Brown, G., Evans, Iii B. M., First principles approach to the magneto caloric effect: Application toNi2MnGa. Journal of Applied Physics, 2011. 109(7): p. 07A942.

41. D.M. Nicholson, K.O., B.A. Shassere, O. Rios, J. Hodges, G.M. Ludtka, W.D. Porter, A.S. Sefat, A. Rusanu, B.M. Evans III. Modeling and Characterization of the Magnetocaloric Effect in Ni2MnGa Materials. in Fifth IIF-IIR International conference in magnetic refrigeration at room temperature, Thermag V. 2012. Grenoble, France.

42. Kabsch, W., Journal Applied Crystallography, 1993. 26: p. 795-800.

43. Hammersley A.P., S.S.O., Hanfland M., Fitch A.N., Hausermann D., High Pressure Research 1996. 14: p. 235-248.

44. $\quad$ Chung, J.S., Ice, G.E., Automated indexing for texture and strain measurement with broad-bandpass x-ray microbeams. Journal of Applied Physics, 1999. 86(9).

45. Barabash, R.I., Ice, G.E., ed. Strain and Dislocation gradients from diffraction. 1st ed. 2014, Imperial College Press: London. 463.

46. Faran, E. and D. Shilo, The kinetic relation for twin wall motion in NiMnGa-part 2. Journal of the Mechanics and Physics of Solids, 2013. 61(3): p. 726-741. 


\section{Figure Captions}

Fig. 1 SEM images demonstrate multiscale character of the twinned structure of the NiMnGaFeCu alloy.

Fig. 2 Compatible TB (a) and an enlarged region of the boundary (b) show continuity at the TB at the nanoscale. Twin colonies at different magnification show that the average thickness of the twin lamellae within the colony is below $100 \mathrm{~nm}(\mathrm{c}, \mathrm{d})$.

Fig. 3. Left: observed reciprocal space projected onto (001) plane of the twin orientations. Circles denote reciprocal vectors, and numbers denote 1 indices of observed reflections from orientation 1 (green), 2 (magenta) and overlapping reflections from both lattice orientations (black). $\boldsymbol{a}^{*}$ and $\boldsymbol{b}^{*}$ are basic vectors of the reciprocal lattices of orientation 1 (green), 2 (magenta) and both lattices in the pseudo orthorhombic setting (black). Basic vectors in the pseudo orthrhombic setting are shown the same for both lattices because angular shift between the orientations in this setting is very small: $\sim 0.3^{\circ}$. Right: relations of basic vectors of the orientations 1 (green) and 2 (magenta) with their basic vectors in the pseudo-orthorhombic setting (black) and basic vectors of $\mathrm{L} 22_{1}$-like lattice (brown). $\boldsymbol{a}$ vecors of both lattices coincide with their $\boldsymbol{- a}$ in the pseudo-orthorhombic setting.

Fig. 4. Typical Laue patterns from a single crystal of $\mathrm{NiMnGaFeCu}$ collected at two angular positions of the sample shifted from one another by $9^{\circ}$. Reflections of twined orientation 1 are marked by green and those of the orientation 2 are marked by magenta. Precise predicted positions of selected reflections are shown on incertions. Predicted positions of reflections (7 3 2), (4 - 12 ) from orientation 1 and (7 3 -2), (2 0 -1) from orientation 2 are shown together with 
their predicted positions from the opposite orinetation reflected in the plane of twinning (denoted by the corresponding color). Splitted reflections are denoted by white rectangles. 

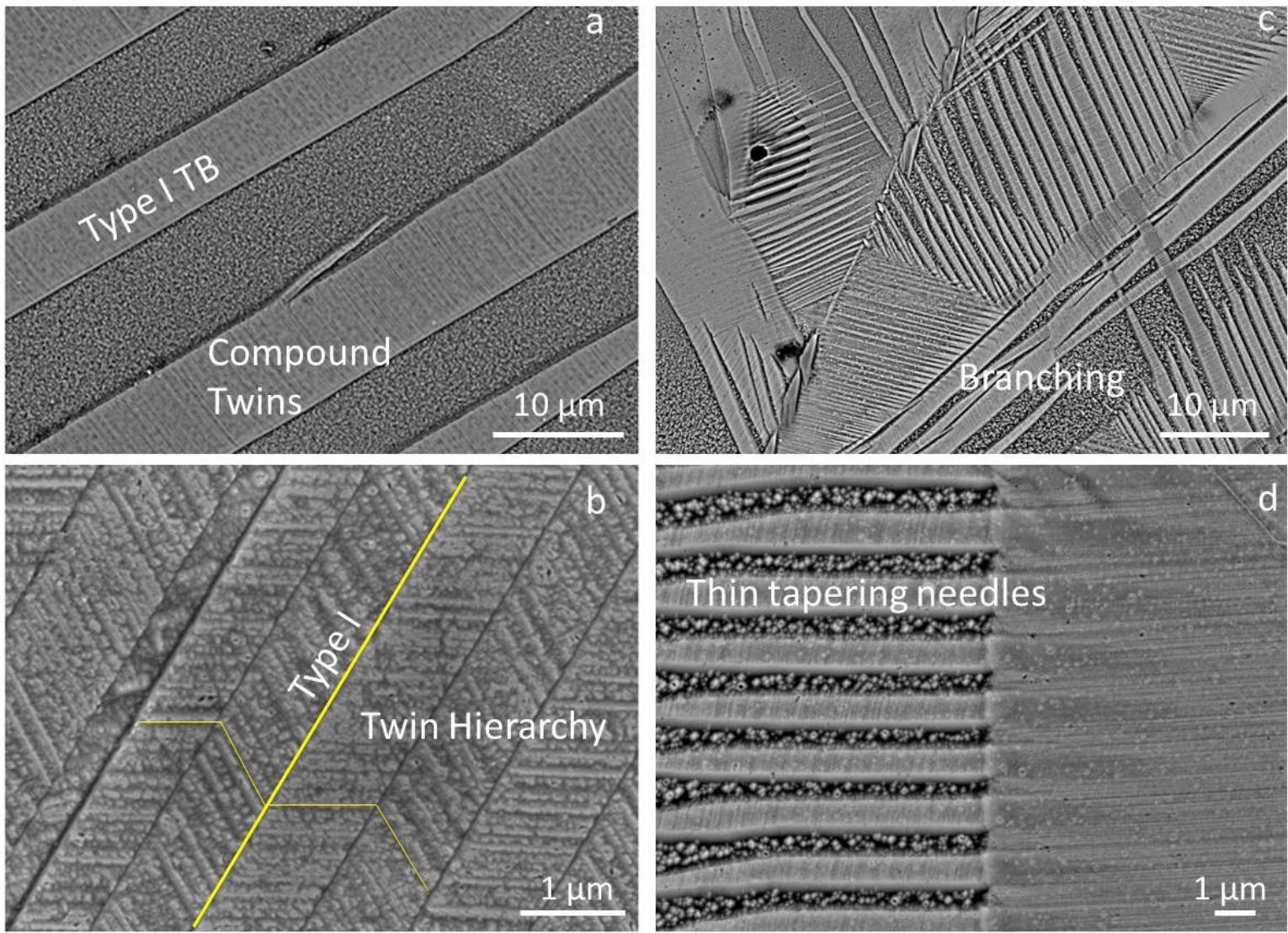

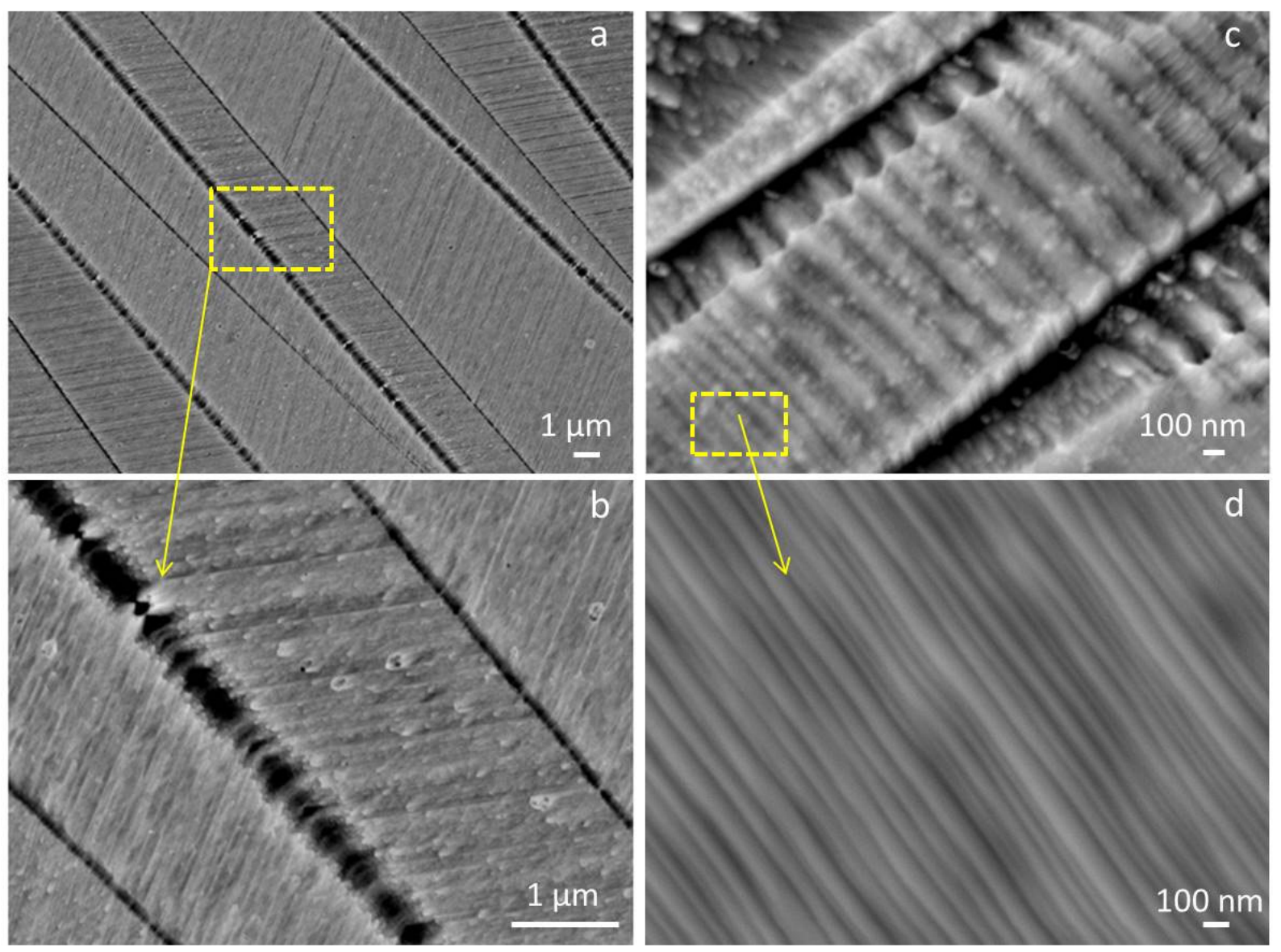


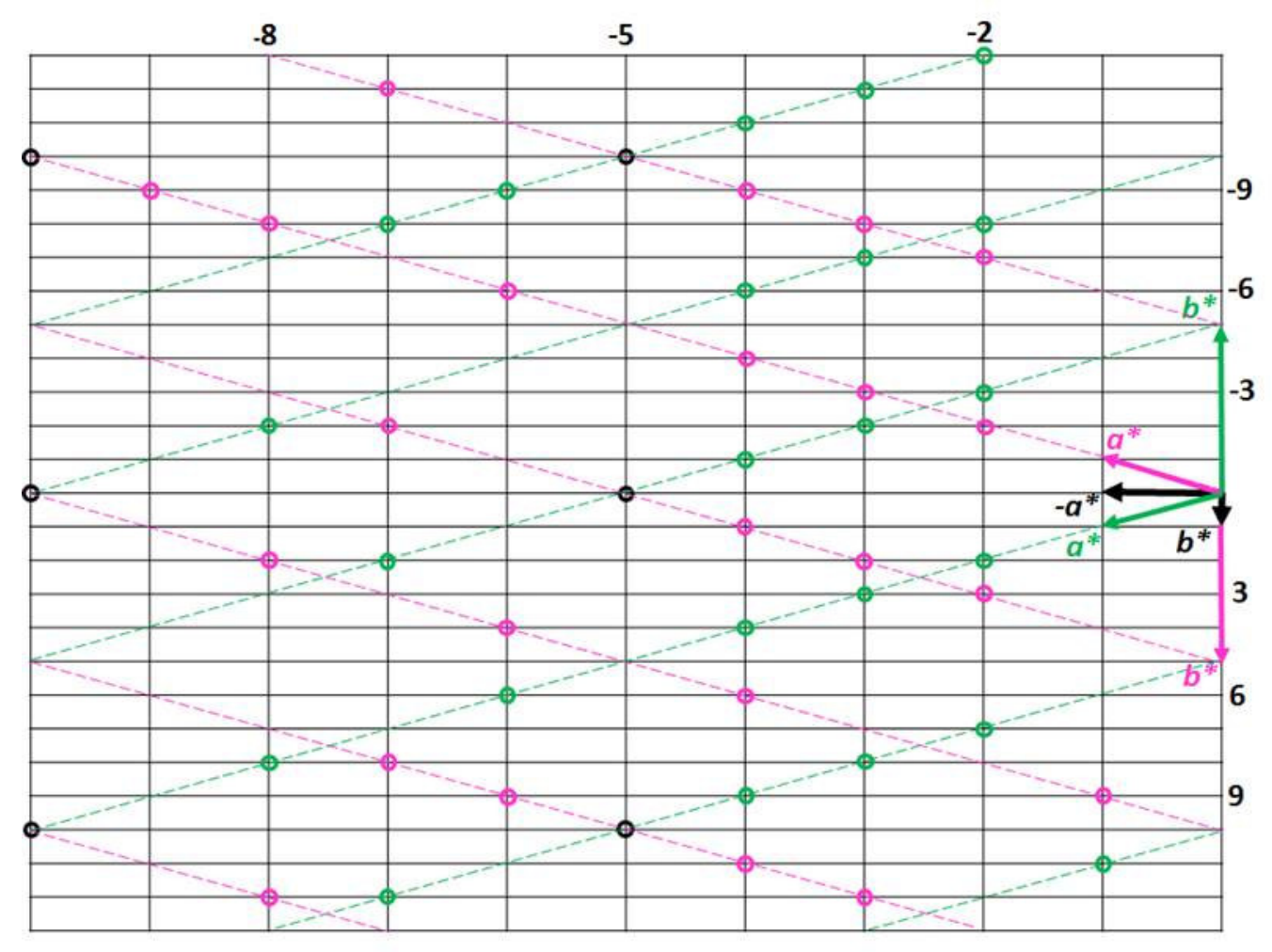

a

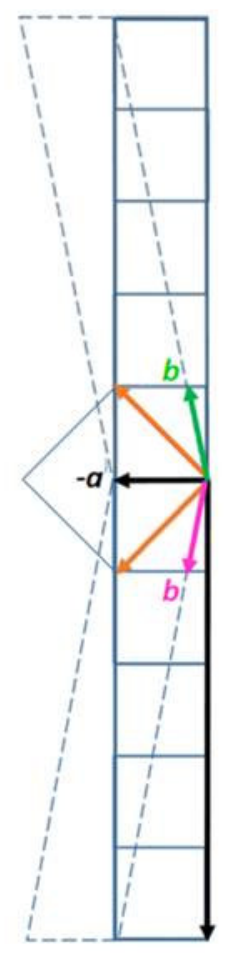

b 


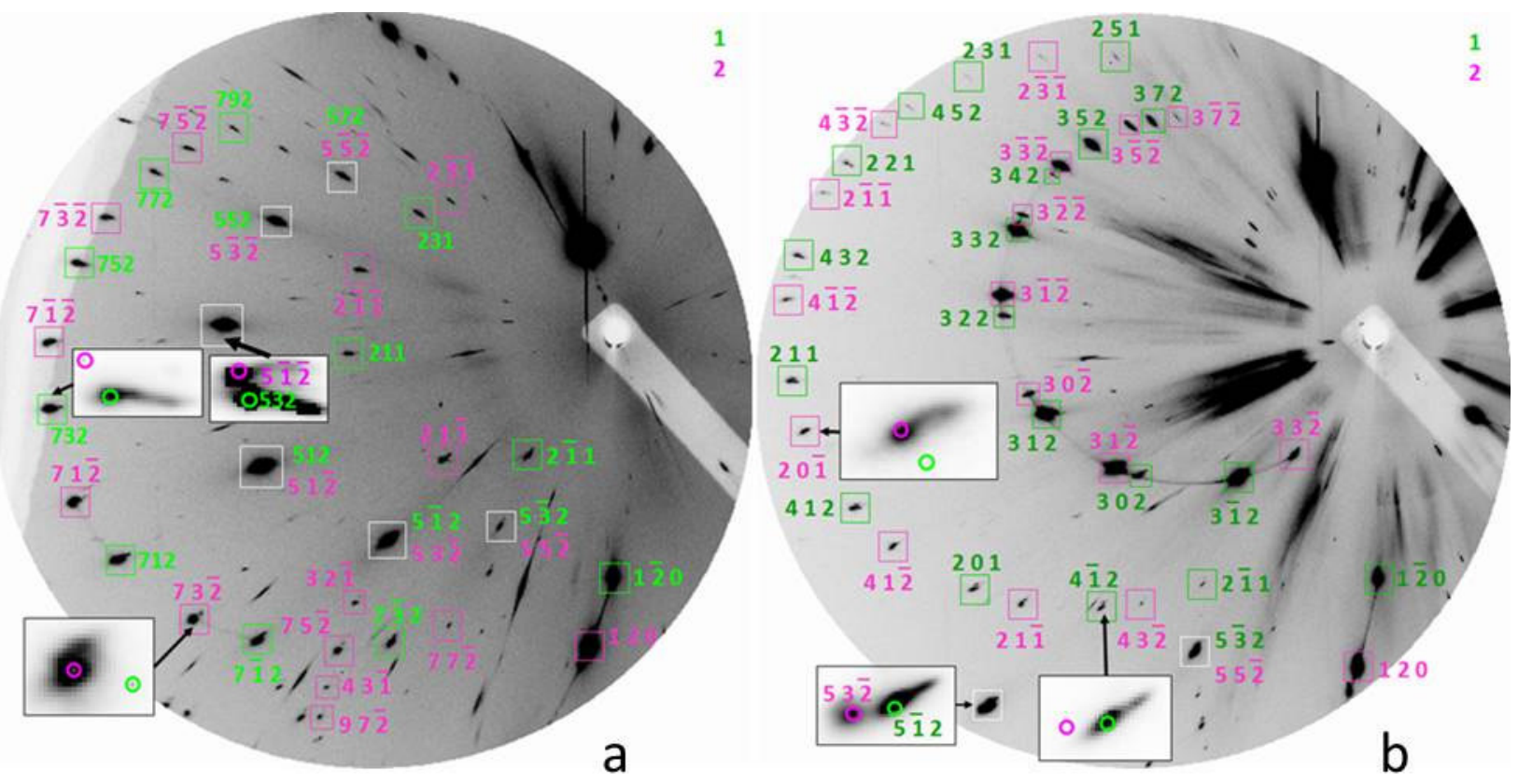

